\title{
loT-based Smart Meeting Room Weather Detection System using Arduino and Relative Sensors
}

\author{
Ahmed Al Marouf \\ Department of CSE \\ Daffodil International University \\ Dhaka-1207, Bangladesh
}

\author{
Shariful Islam \\ Department of CSE \\ Daffodil International University \\ Dhaka-1207, Bangladesh
}

\author{
Narayan R. Chakraborty \\ Department of CSE \\ Daffodil International University \\ Dhaka-1207, Bangladesh
}

\begin{abstract}
Internet of Things (IoT) has become an emerging area of research as the interaction between hardware and software components have become easier nowadays. Different IoT solutions are designed, prototyped, implemented and commercialized in this millennium, such as home automation systems, smart locker systems, smart city connectivity etc. On the eve of 4th Industrial revolution, the demand of smart automated systems for solving house-hold problems and commercial products has increased. Therefore, in this paper, we have proposed an IoT-based smart meeting room weather detection system, which will enable to give the real-time situation of a meeting room. Smart meeting room are nowadays incorporating smart table-top devices, smart boards, projectors and/or ergonomically healthy seating arrangements. However, the idea of healthy environment in meeting rooms could be ensured by incorporating reliable sensors. For our proposed system, we have used temperature and humidity sensor (DHT22), barometric pressure sensor (BMP180) and gas sensor (MQ135). For communication, we have used the Bluetooth module (Hc-05) and for giving alerts a buzzer has been utilized. The proposed system can be used to convert and ordinary room to smart meeting room, in terms of weather condition. Based on the reading of sensors, a smart system has been proposed to maintain a healthy environment of meeting room. The overall criteria are formalized and users may use the mobile application to get accessed the sensor data.
\end{abstract}

\section{General Terms}

Internet of Things (IoT), Arduino, Mobile Application.

\section{Keywords}

Internet of Things (IoT), Sensors, Smart Meeting Room, Weather detection, Mobile Communication.

\section{INTRODUCTION}

In the era of modern science and technology, Internet of Things (IoT) has become a booming research topic to be explored. The connectivity between things and devices has come to the melting point to minimize the gap between the devices and human. Designing interfaces for human computer interaction (HCI) based solutions has become complex due to the integration. Though the diversity of using sensors to understand and evaluate the environment has evolved to a new dimension. Because of the increased capability of artificial intelligence, the gap between the sensors and its interpretation in software solutions has become narrower. The use of mobile based sensors has also increased because of built-in sensors in the smart phones. As the world progressed in digital form, the demand of robots or mobile based interaction with the hardware has increased, which reflected in the involvement of IoT devices in household works. Nowadays, the cleaner robot [1] could be operated from anywhere anytime. Smart home automation systems [2-5] enables the access to on/off the lights, fans, air conditioners and many more household devices from far away using mobile applications. Different IoT based solution such as smart locker systems and parcel delivery method [6], smart city [7-10] etc. has not only gained popularity because of design and innovativeness, but also gained demand for commercialization.

Nowadays, smart meeting rooms are incorporating various smart devices such as table top devices for taking instant notes and showing demonstrations, smart boards for drawing without any use of ink, hanged projectors for video or interactive presentations and ergonomically healthy chairs or seating arrangements. All these different smart utilities are for better involvement of human minds towards the main agenda of the meetings and increase productivity. Apart from all these necessary things, one of the main aspect of a meeting room is the environment of the room. The temperature, humidity level, gas contamination on the air, the air pressure level are necessary components to look after in a meeting room for healthy environment. It is evident, that the air pressure has direct impact on human psychology and people are affected by the temperature and humidity level seriously. The contamination of different gases on air could cause serious damage in environment. Therefore, integrating these parameters into a smart system could give better system.

On that perspective, we have developed an IoT-based smart meeting room weather detection system using Arduino and relative sensors. We have incorporated the temperature and humidity sensor, gas sensor, barometric air pressure sensor for capturing the temperature, humidity, gas contamination and air pressure, respectively. The most essential component of an IoT system is the communication over internet from the device. The Bluetooth facility of smartphones could be a minimal connectivity for device and over internet the mobile applications data could be referred to any device having internet connections. An alert using strong buzzer could be utilized to give notification on the risky situation.

The rest of the paper contains section II describing the background study of related IoT systems. In section III, we have presented the proposed system architecture and section IV consists the description of the hardware used for the proposed system (sensors and system boards). The software design, circuit design and implementation are presented in section V. The section VI includes the experimental results using mobile application and the section VI concludes the paper with some future scopes. 


\section{BACKGROUND STUDY}

In this section, we have formalized the related works previously performed by many researchers to solve the similar problem. This section is mainly divided into several parts consisting the hardware and software tools used to make an ordinary room into a smart room; smart meeting room facilities [11] and computer vision based intelligent systems to track activities such as writer identification [12].

In [11] A. Waibel et. al. presented a smart meeting room task (SMaRT) performed in Interactive Systems Laboratories (ISL). The system includes the pre-meeting activities such as calling meeting participants, navigating the participants to the meeting venue, creating a meeting condition in terms of atmosphere etc.; in-meeting activities such as presentation facilities, changing presentation slides, providing on demand information to all etc. and the post-meeting activities like indexing and archiving the meeting minutes using video and audio recordings. This paper also focused on the people tracking and identification techniques, speaker, accent and language identification techniques. Finally, a system architecture named SMaRT [11] has been introduced for the task.

An artificial intelligence based research institute name IDIAP has proposed a smart meeting room [12] having dimensions as $8.2 \mathrm{~m} \times 3.6 \mathrm{~m} \times 2.4 \mathrm{~m}$ rectangle room having ample digital facilities. The primary purpose of the meeting room is to capturing visual, audio and textual data within the meeting scenarios. Different modules of hardware had been used for audio acquisition and video capturing. "The current configuration uses three cameras with wide-angle lenses, six lapel phones and two 80element microphone arrays to record meetings containing up to six participants [12]".

Using the same meeting room proposed in [12] researchers have worked on identifying the writer on the board in [13]. A Gaussian Mixture Model (GMM) based approach has been adopted in [13] to recognize the writer on the board using the smart meeting room.

A structure of detecting smart meeting room using pervasive computing technologies has been presented in [14]. The research presented a detection system of starting and ending of the meeting \& provides support to the participants in context information, knowledge usability and ephemeral group communication [14]. The paper considered four practical scenarios to propose classification of meeting using state diagrams and identifying the starting time and ending time using proposed algorithms. But, the system lacks the degree of fault-tolerance, scalability and security concerns [14] in the proposed architecture.

Smart meeting rooms could be utilized in research problems such as human tracking, head tracking and action recognition [15] etc. H. Nait-Charif et. al. [15] proposed an action recognition and head tracking system utilizing PETS-ICVS 2003 video dataset, which originally developed for evaluating smart meeting applications. The proposed method used the Iterated Likelihood Weighting (ILW) and Sampling Importance Resampling (SIR) methods for precise detection of head tracking and specific actions.

A survey on smart meeting systems has been conducted by $\mathrm{Z}$. $\mathrm{Yu}$ and Y. Naramura in [16] which incorporates the state-ofthe-art methods and open issues found in this context. A generic architecture of smart meeting room has been proposed in [16] having semantic processing, meeting recognition and meeting capture modules. The meeting recognition consists person identification and speech detection methods while the meeting capture module comprises of video, audio and some contextual issues like room layout, meeting agenda, seat positions, emotions, locations and whiteboard notes. These data could be captured using different types of sensors (e. g. head-tracking sensors, physiological sensors, lighting sensors, pressure sensors, RFID, pen strokes etc.)

The internal atmosphere of the meeting room has directly impacts on the mind of the participants. It is evident that, the participants get distracted while there is a presence of unusual objects (not related to meeting or even food) and/or unwanted company. In this paper, we have focused on the overall environment of smart meeting room. We have used several sensors to capture the environmental condition of the meeting room and detect if the room is compatible to be considered as a smart meeting room. The overall atmospheric condition of the room could be understood from the gas contamination in the air, the temperature level, humidity level and air pressure level of the room. All the parameters are directly concern about the health issues of the participants.

In this paper, we have proposed a detection of weather condition for a smart meeting room comprising the sensors to get real-time values and detect if the room condition is good enough for a smooth meeting conduction.

\section{SYSTEM ARCHITECTURE}

In this section, we have presented the architecture of the proposed system. The system diagram shows the main components used in the proposed system. The fig. 1 shows the system architecture which consists the LCD display, gas sensor, temperature and humidity sensor, pressure sensor, a buzzer and everything is connected to the Arduino Mega board.

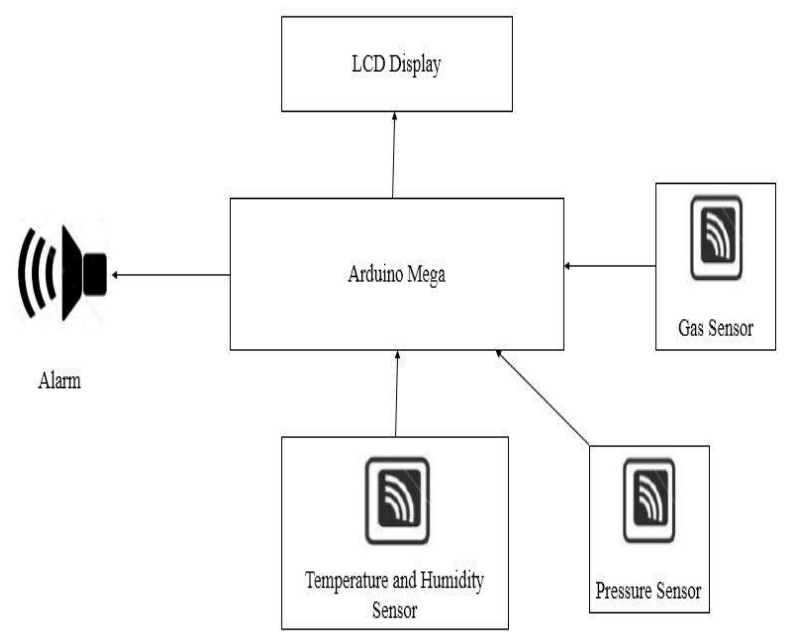

Fig. 1. Proposed System Architecture.

The proposed system has three input sensors and two output medium. Three input sensors are temperature and humidity, gas and pressure sensors $\&$ the output medium are the alarm system and the LCD display. The input and output components are connected with the Arduino Mega. The elaboration of the proposed system have been described in section IV and section V, consisting the components needed and the software design, respectively. 


\section{HARDWARE COMPONENTS}

In this section, we have briefly described the hardware components that we have utilized for designing the proposed system. The section is divided into part for describing the components.

\subsection{Arduino Uno}

Arduino is a prototype platform for electronics that many use as a way to learn about digital electronics. It provides easy access to a whole set of knowledge. One of the key features of this technology is that it was designed with users in mind from the beginning. The Arduino UNO is an open - source microcontroller board evolved by Arduino.cc and based on the Microchip ATmega328P module. The board has sets of digital and analog input / output (I / O) pins that can be interfaced with various boards of expansion (shields) and other circuits [17]. The Arduino UNO board includes 6 analog pins, 16 digital pins and programmable with the Arduino IDE using type B USB cable. The Arduino IDE is an interactive IDE which takes the codes written as source code, which deals with the sensors connected with the single microcontroller board. Fig 2. shows the Arduino Uno board.

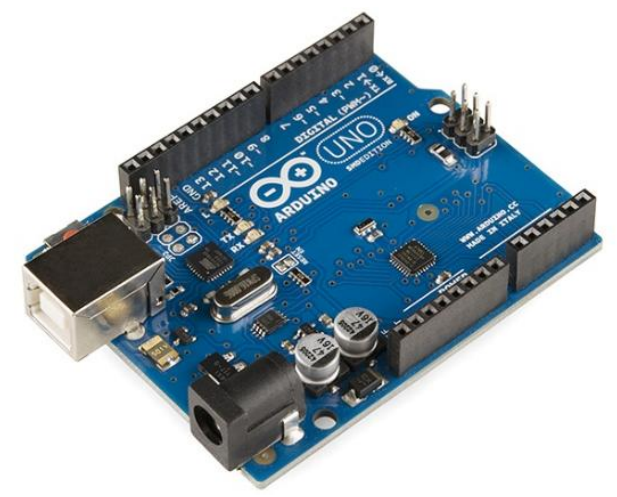

Fig. 2. Arduino UNO [18]

\subsection{Bluetooth Module}

"HC-05 Bluetooth Module is an easy to use Bluetooth SPP (Serial Port Protocol) module, designed for transparent wireless serial connection setup. Its communication is via serial communication which makes an easy way to interface with controller or PC. HC-05 Bluetooth module provides switching mode between master and slave mode which means it able to use neither receiving nor transmitting data [19]".
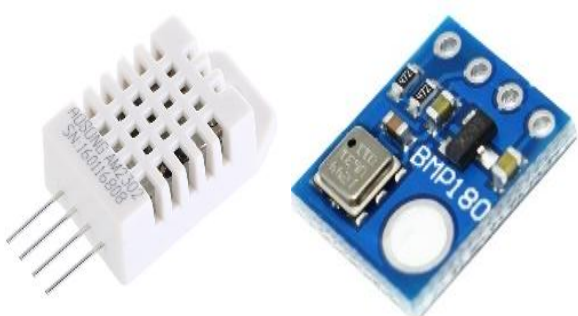

Fig. 3. HC-05 Bluetooth Module [19]

\subsection{Temperature \& Humidity Sensor}

"The DHT22 is a basic, low-cost digital temperature and humidity sensor. It uses a capacitive humidity sensor and a thermistor to measure the surrounding air, and spits out a digital signal on the data pin (no analog input pins needed). It's fairly simple to use, but requires careful timing to grab data. The only real downside of this sensor is you can only get new data from it once every 2 seconds, so when using our library, sensor readings can be up to 2 seconds old [20]."

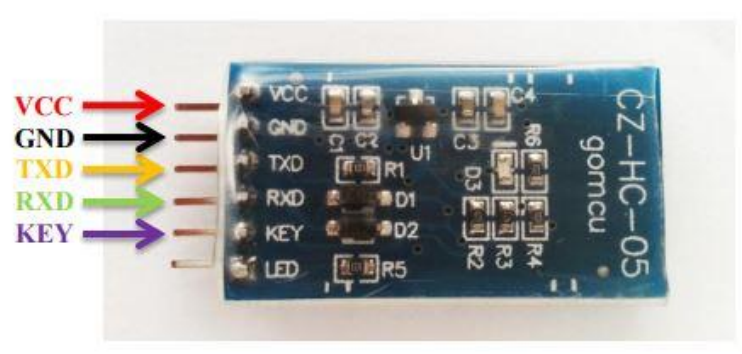

Fig.4. DHT-22 Temperature and Humidity Sensor [20] \& BMP-180 Barometric Pressure Sensor

\subsection{Barometric Pressure Sensor}

A low cost sensing solution for measuring barometric pressure and temperature is BMP 180 which is developed by Bosch. The sensor is soldered onto a PCB with a $3.3 \mathrm{~V}$ regulator, $\mathrm{I} 2 \mathrm{C}$ level shifter and pull-up resistors on the I2C pins. The BMP180 is the next-generation of sensors, and replaces the BMP085. The advantage of using this sensor is that it is completely similar to the BMP085 in terms of firmware/software. The XCLR pin is not physically present on the BMP180 so if we need to know that data is ready we will need to ask the $\mathrm{I} 2 \mathrm{C}$ bus. Along with the temperature module, the air pressure could be measured in mili bar (mb).

\subsection{Gas Sensor}

To recognize the contamination of several gases in the air, the MQ 135 gas sensor may work precisely. It can detect the hazardous gases such s ammonia, $\mathrm{CO}_{2}$, aromatic compounds, sulfur, benzene vapor, smoke and other harmful gases in fixed or closed environment. The sensor is ideal to be used in office or factory as it contains simple drive and monitoring circuit.

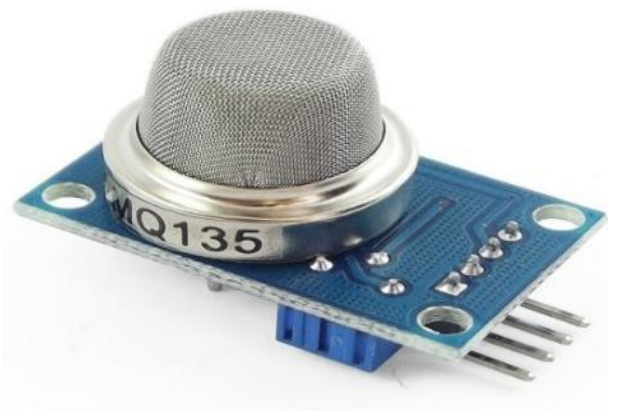

Fig. 5. MQ 135 Gas Sensor

\subsection{Other Accessories}

The other accessories needed to design the whole circuit module are LCD display, solder Vero board, buzzer, potentiometer and connecting wires. The detail descriptions of these accessories could be found in the manuals of the products, respectively. The Fig. 6 illustrates these accessories for the visual interpretation. 


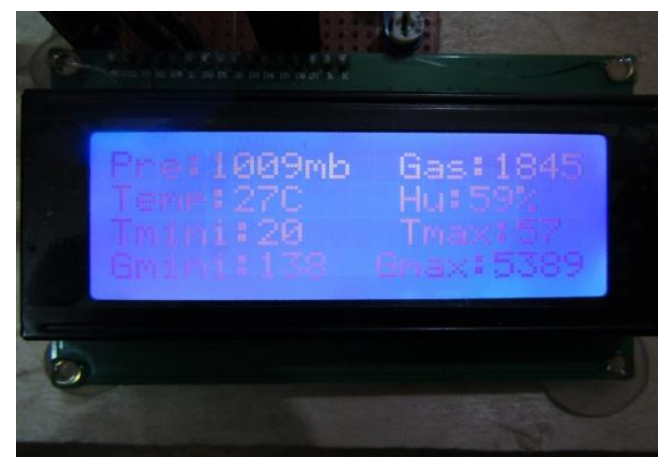

(a)

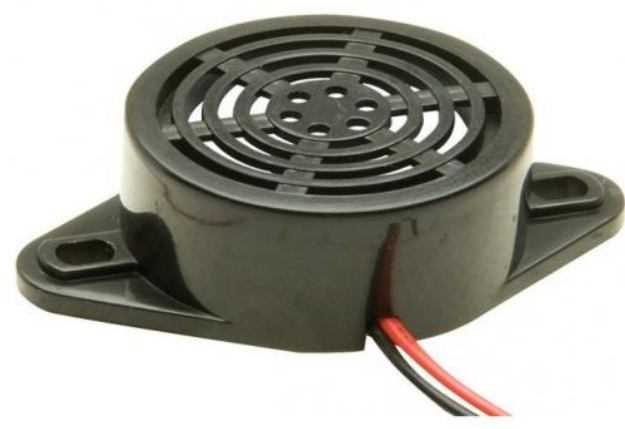

(c)

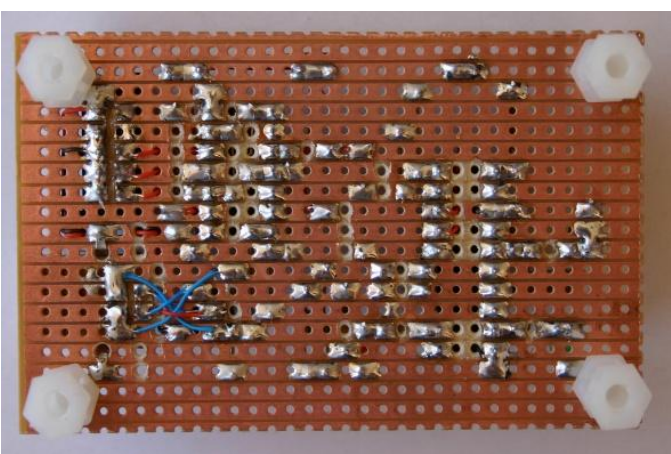

(b)

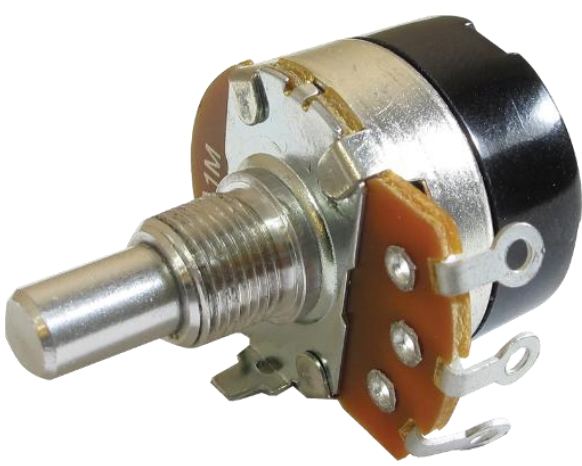

(d)

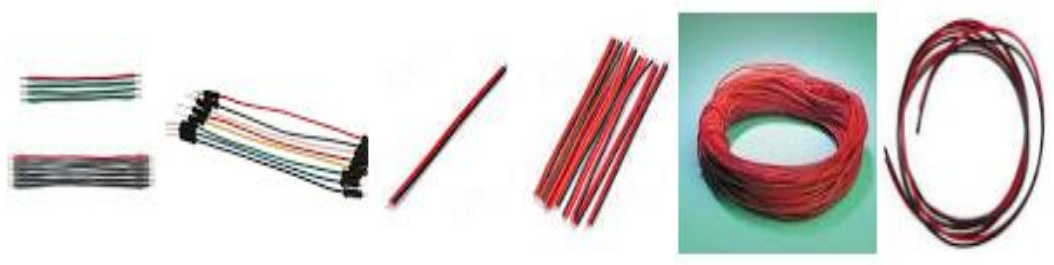

(e)

Fig. 6. Visual Illustration of accessories (a) LCD Display, (b) Solder Vero Board, (c) buzzer, (d) potentiometer and (e) connecting wires.

\section{SOFTWARE DESIGN AND INPLEMENTATION}

This section demonstrates the software design and implementation of the total proposed system. The section is divided in to four major parts: software implementation of circuit design using Proteus Software; implementation of the design using Arduino IDE, testing the system using PuTTY computer monitoring and mobile application.

\subsection{Circuit Design}

For circuit design purpose, there are several software tools which may become handy. The circuit design contains all the hardware components mentioned in section 4 . The design has been performed and formalized in Proteus. The Proteus Design Suite [21] software is used for circuit simulation and PCB design. The ease of using schematic capture, firmware IDE and PCB layout on a single platform makes it a complete software solution. This proprietary software tool is used primarily for electronic design automation. The software could be utilized by electronic design engineers and technicians to design schematics and manufacturing printed circuit boards. The main reason of using this software for our experimentations is the flexibility and parallel development of both the system hardware and system firmware. The reduced time for testing the design with several configuration is another reason. The detail circuit design has been depicted in Fig. 7.

\subsection{System Implementation}

For the implementation of the system we have utilized the Arduino IDE, as we have used the Arduino Uno as the system board for our proposed design. We have connected the separate modules with Arduino Uno suing the connecting wires. And the implementation has been tested using several platforms such as Arduino Serial monitor and PuTTY. The proposed system is tested with several scenarios such as in smoke, the gas sensor gives higher values. Similarly the change in air pressure \& temperature up-down are monitored using the proposed system. The sensor dependent system acts as the sensors starts to give unwanted or alarming data values. 

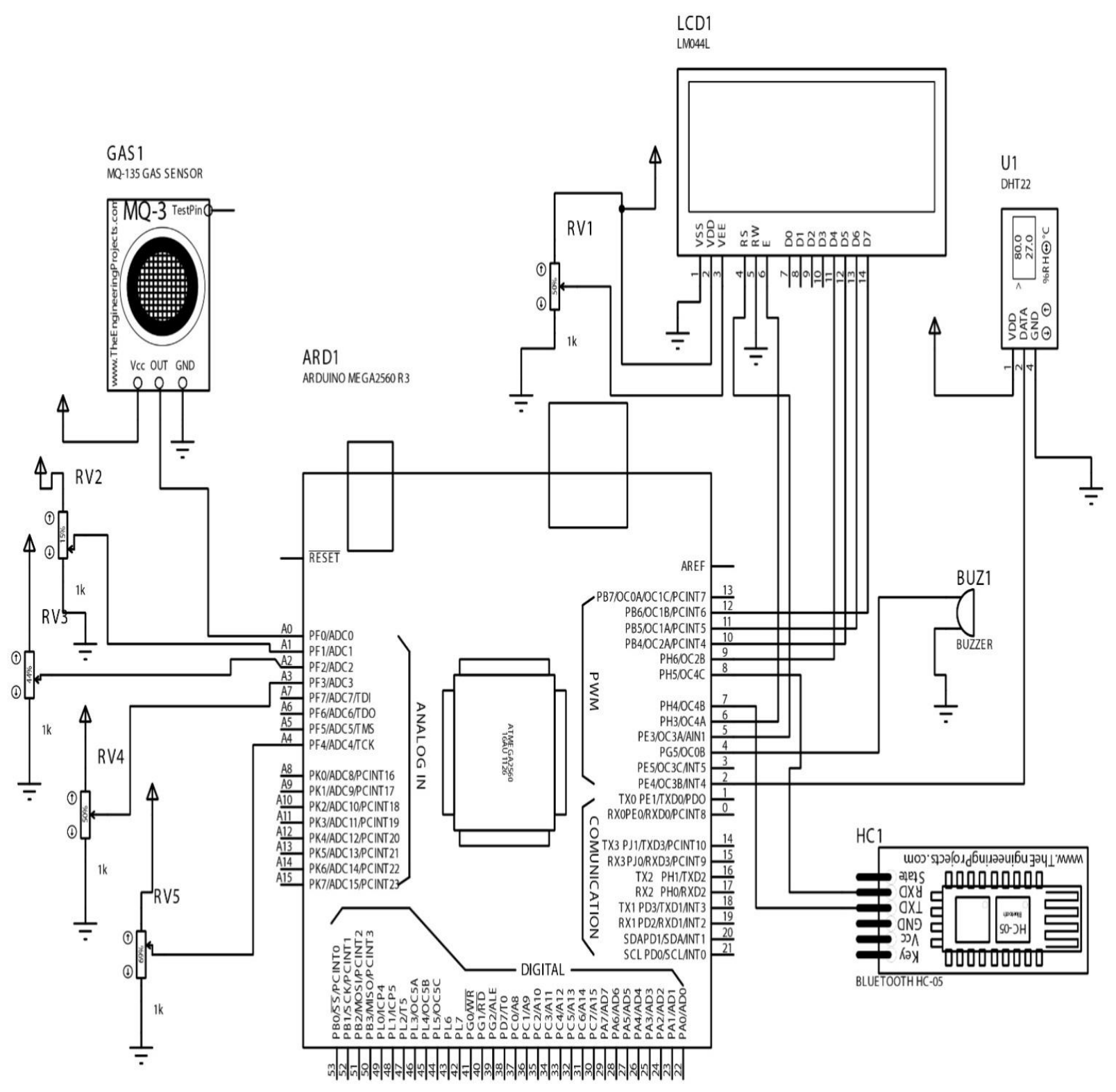

Fig. 7. Circuit Design (Developed in Proteus Design Suite) 


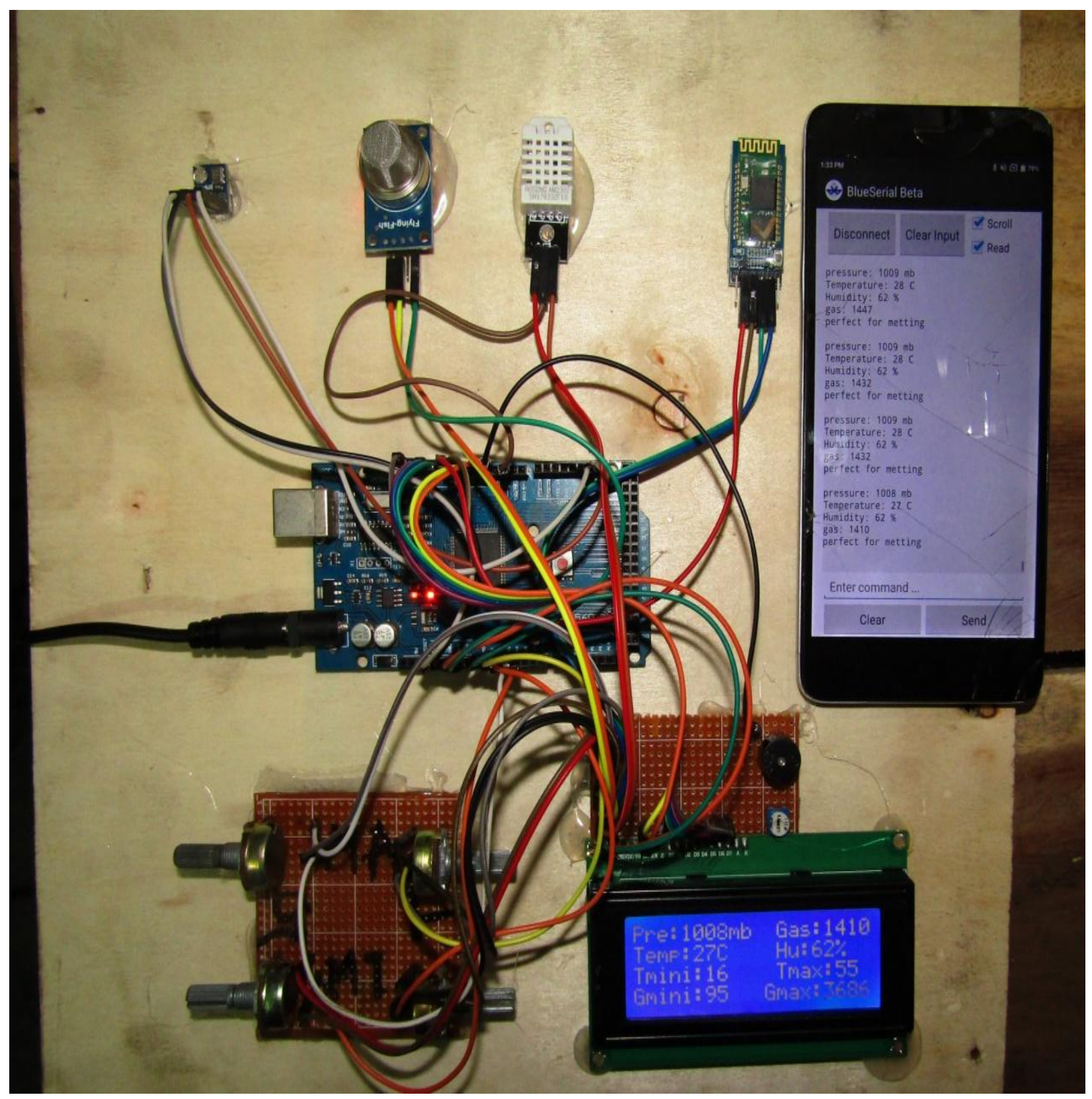

Fig. 8. Implementation of Proposed System

The Fig 8 shows the total implementation of the proposed smart meeting room weather detection system. The sensors as depicted in Fig 6 are connected using wires to implement the same design of Fig 7.

As the mobile application is connected with the Bluetooth sensor, the serial communication gives the sensor data from the system after every 30 seconds. The LCD display showing the same results and updates the values after the same 30 seconds. The LCD display values and the mobile application values are calibrated on the same time. The potentiometers are used to change the voltage to the circuits and some sensors fluctuates as accordingly. The system development needed two Vero boards to connect the potentiometers and LCD display on the same circuit design.

\subsection{System Testing using PuTTy}

PuTTY is an open-source and cost free terminal emulation application. The main advantage of using this serial console is that it supports several network protocols, including SCP, $\mathrm{SSH}$, raw socket connection and telnet.

Because of the easier communication medium researchers are using this application for troubleshooting and maintaining the communication between the hand-crafted implemented device and computer systems. For our work, we have utilized the same to test if the implemented hardware part is fully functioning or not. We have connected the PuTTY to Bluetooth Com port 4. When we start the system after connecting to computer via Bluetooth, the serial communication is setup and the outcome looks like Fig. 9. The same data are shown in the LCD display so that the data could be matched at a certain time period. As the time frame used for the LCD display and the PuTTY application is been doing over the same time, the pressure, temperature, humidity, gas sensor values are showing the same in both the output devices. There is no calibration needed afterwards. 


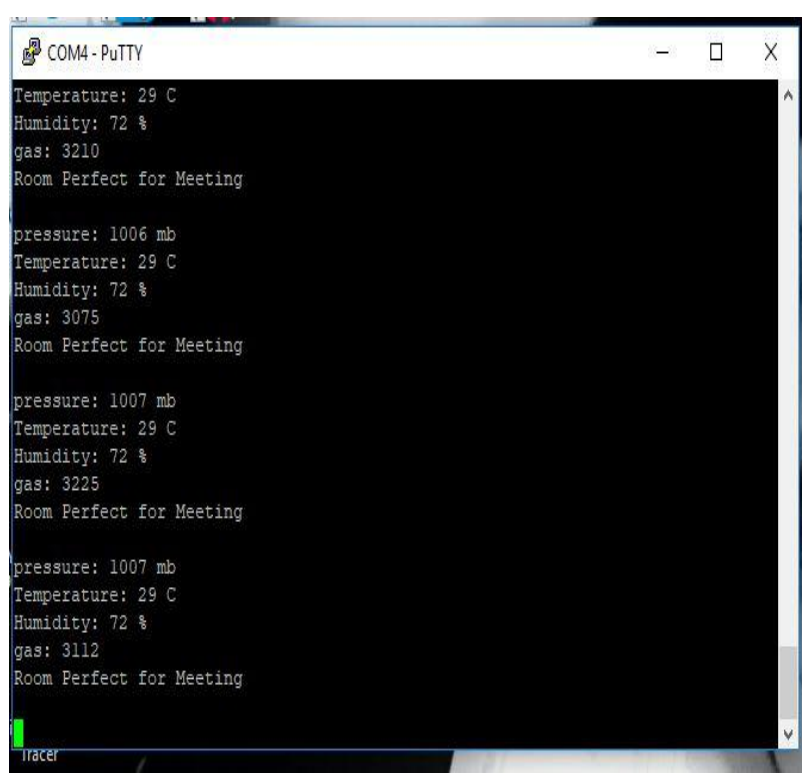

Fig. 9. Serial communication using PuTTY.

\subsection{System Testing using Mobile Apps}

We have also tried to test using Mobile application implementation. The same sensor data are sent to the mobile app for testing. We have collected those data from the mobile application for the further processing in machine learning systems.

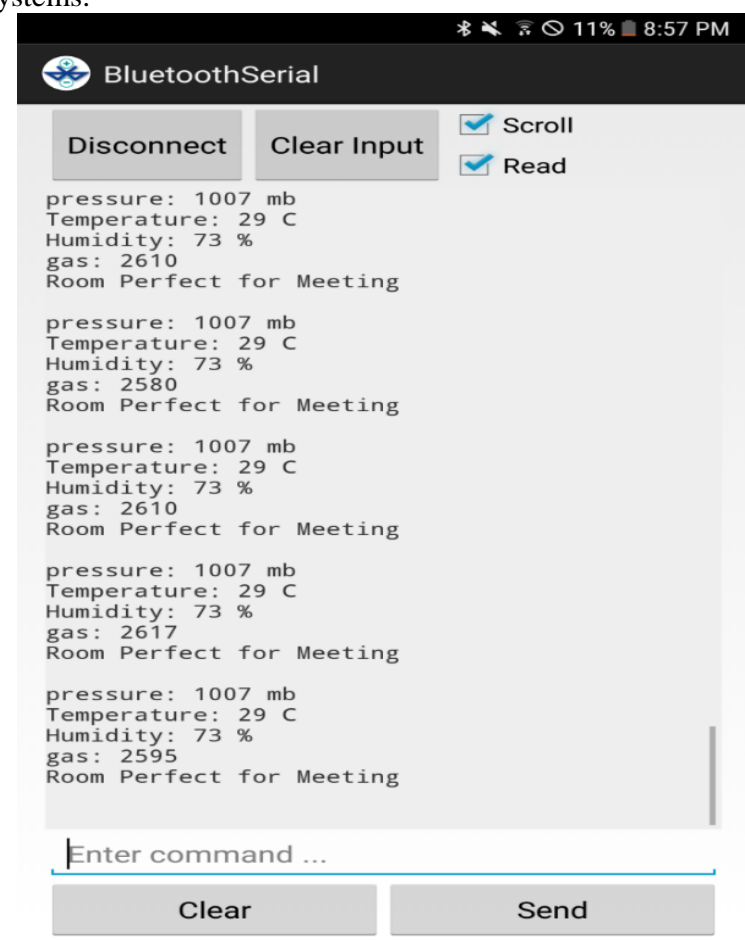

Fig. 10. System testing using Mobile application.

\section{EXPERIMENTAL RESULTS}

We have collected the sensor data coming from the hardware part continuously using mobile application and utilized for building a predictive model based on supervised learning. We have developed an experimental setup using the sensor data for predicting if the smart meeting room is suitable for meeting or not. The experimental model could be depicted from Fig. 11.

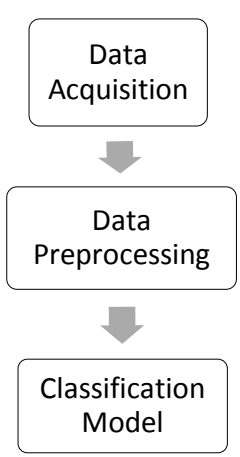

Fig. 11. Experimental method for Predictive system

The sensor data are considered as the features for the supervised learning model and the sent to the next step for data pre-processing. In data pre-processing step, we have tried to find the redundant data and the missing values analysis. Those observations are eliminated for experimentation and passed to the classifiers. For binary classification, we have used five widely used classifiers in the literature namely, naïve bayes $(\mathrm{NB})$, random forest $(\mathrm{RF})$, decision tree (DT), logistic regression (LR) and support vector machine (SVM). The table 1 consists the performance evaluation metrics after applying 10-fold cross validation. All the classifiers have worked fine as the accuracy is in-between $79 \%$ to $90 \%$. SVM has outperformed the other classifiers and gives highest accuracy of approximately $89.98 \%$.

Table 1. Performance Evaluation Metrics of the system

\begin{tabular}{|c|c|c|c|c|}
\hline $\begin{array}{c}\text { Classifier } \\
\text { Name }\end{array}$ & Precision & Recall & F1-score & Accuracy \\
\hline NB & 0.7869 & 0.7915 & 0.7985 & $79.79 \%$ \\
\hline RF & 0.8110 & 0.8113 & 0.8218 & $82.12 \%$ \\
\hline DT & 0.8275 & 0.8220 & 0.8285 & $82.88 \%$ \\
\hline LR & 0.8607 & 0.8527 & 0.8619 & $86.11 \%$ \\
\hline SVM & 0.8975 & 0.8879 & 0.8995 & $\mathbf{8 9 . 9 8 \%}$ \\
\hline
\end{tabular}

\section{CONCLUSION \& FUTURE SCOPES}

In this paper, we have presented the hardware and software contribution for predicting if the smart meeting room is healthy and suitable enough for a meeting. For this binary classification, we have developed a hardware using the necessary sensors such as gas sensor, temperature and humidity sensor, air pressure sensor etc. The hardware is tested using PuTTy and mobile application as well. For experimentation we have utilized the sensor data and fetched them into classification model. For our experimentation we have used five different classification algorithms and SVM outperforms the other existing classifiers. For future works, we may fabricate the hardware into microchips and control it using mobile applications. A similar hardware chip would be useful for integrating into smart phones so that they could recognize the suitable places for meeting at any time anywhere. 


\section{REFERENCES}

[1] JG Song, J Ko, S Moon, K Lee, "Robot cleaner, robot cleaning system and method for controlling same" - US Patent 6,732,826, 2004

[2] R. Teymourzadeh, S. Ahmed, K. W. Chan, and M. V. Hoong, "Smart GSM based home automation system," in Systems, Process Control (ICSPC), 2013 IEEE Conference on, Dec 2013, pp. 306-309.

[3] H. Elkamchouchi and A. ElShafee, "Design and prototype implementation of sms based home automation system," in Electronics Design, Systems and Applications (ICEDSA), 2012 IEEE International Conference on, Nov 2012, pp. 162-167.

[4] B. Ghazal, M. Kherfan, K. Chahine, and K. Elkhatib, "Multi control chandelier operations using xbee for home automation," in Technological Advances in Electrical, Electronics and Computer Engineering (TAEECE).

[5] B. H. Sunil, "Household security system based on ultrasonic sensor technology with SMS notification," European Journal of Academic Essays, vol. 1, no. 4, pp. 6-9, 2014.

[6] L Torres, B Suggs, "Smart locker system and method of parcel delivery" US Patent App. 14/582,088, 2015.

[7] Albino, V., Berardi, U., \& Dangelico, R. (2015). Smart cities: Definitions, dimensions, performance, and initiatives. Journal of Urban Technology, 22(1), 3-21. http://dx.doi.org/10.1080/10630732.2014.942092.

[8] S. Alawadhi, A. Aldama-Nalda, H. Chourabi, J.R. GilGarcia, S. Leung, S. Mellouli, T. Nam, T.A. Pardo, H.J. Scholl, S. Walker, "Building Understanding of Smart City Initiatives," Lecture Notes in Computer Science 7443 (2012) 40-53.

[9] S. Allwinkle, and P. Cruickshank, "Creating Smarter Cities: An Overview," Journal of Urban Technology 18: 2 (2011) 1-16.

[10] P. Neirotti, A. De Marco, A.C. Cagliano, G. Mangano, and F. Scorrano, "Current Trends in Smart City Initiatives: Some Stylised Facts," Cities 38 (2014) 2536.

[11] A. Waibel, T. Schultz, M. Bett, M. Denecke, R. Malkin, I. Rogina, R. Stiefelhagen, and J. Yang, "SMaRT: the smart meeting room task at isl," in IEEE Int'l. Conf. Acoustics, Speech, and Signal Processing, vol. 4, 2003, pp. $752-755$

[12] D. C Moore, "The IDIAP Smart Meeting Room", IDIAPCOM, 02-07, November 2002.

[13] M. Liwicki, A. Schlapbach, H. Bunke, S. Bengio, J. Mariethoz and J. Richiardi,"Writer Identification for Smart Meeting Room Systems", International Workshop on Document Analysis Systems, DAS 2006: Document Analysis Systems VII pp 186-195.

[14] S. Ahmed, M. Sharmin, S.I. Ahamed, "A smart meeting room with pervasive computing technologies", Sixth International Conference on Software Engineering, Artificial Intelligence, Networking and Parallel/Distributed Computing and First ACIS International Workshop on Self-Assembling Wireless Network, 23-25 May 2005, Towson, MD, USA, USA.

[15] H. Nait-Charif \& S. J. McKenna, "Head Tracking and Action Recognition in a Smart Meeting Room", IEEE International Workshop on Performance Evaluation of Tracking and Surveillance, Graz,, Austria, 31 March 2003.

[16] Z. Yu, Y. Nakamura, "Smart meeting systems: A survey of state-of-the-art and open issues", Journal ACM Computing Surveys (CSUR) Surveys Homepage archive Volume 42 Issue 2, February 2010.

[17] Adruino UNO for beginners - Progects, Programming and Parts". makerspaces.com. Retrieved 4 February 2018.

[18] Arduino Uno Visuals, Available [Online] https://en.wikipedia.org/wiki/Arduino_Uno\#/media/File: Arduino_Uno_-_R3.jpg

[19] Bluetooth Module, Available [Online] https://www.gme.cz/data/attachments/dsh.772-148.1.pdf

[20] Temperature and Humidity Sensor, Available [Online] https://cityos-air.readme.io/docs/4-dht22-digitaltemperature-humidity-sensor

[21] Proteus Software, Available [Online] https://www.labcenter.com/ 